\title{
Patientensicherheits-Benchmarking in Schweizer Spitälern
}

\author{
Die Stiftung für Patientensicherheit führte in den Jahren 2008 und 2009 erstmals \\ eine Benchmarking-Studie mit acht Deutschschweizer Spitälern durch. Ziel war, die \\ von Patienten während des stationären Aufenthalts beobachteten Fehler systema- \\ tisch zu erfassen und zu analysieren.
}

\section{Olga Frank, Marc-Anton Hochreutener, David Schwappach}

Lesen Sie hierzu auch das «Zu guter Letzt» auf Seite 378 .

\footnotetext{
* Die Literaturangaben finden sich im Internet unter www.saez.ch $\rightarrow$ aktuelle Ausgabe oder $\rightarrow$ Archiv $\rightarrow 2010 \rightarrow 9$
}

Interessenbindungen:

Die Benchmarking-Studie wurde vom Bundesamt für Gesundheit (BAG) finanziell unterstützt. Das BAG nahm keinerlei Einfluss auf das Studiendesign, den gesamthaften BenchmarkingProzess und die Publikation von Ergebnissen.

Korrespondenz: Dr. Olga Frank Stiftung für Patientensicherheit Asylstrasse 77 CH-8032 Zürich Tel. 0432437670 Fax 0432437671

frank@patientensicherheit.ch

\section{Einleitung}

Als Grundlage für das klinische Risikomanagement gewinnt die Messung der Patientensicherheit zunehmend an Bedeutung. Neben expertenorientierten Ansätzen ist die Wissensressource «Patient» eine für die Gesundheitseinrichtungen wichtige Quelle zur Beurteilung der Patientensicherheit.

Aber - sind Patienten überhaupt in der Lage, Fehler während der medizinischen Behandlung zu beobachten und sicherheitsrelevante Aspekte zu erkennen? Wenn ja, könnten Auskünfte der Patienten dazu beitragen, Fehler zu vermeiden!

Die Diskussion darüber wird kontrovers geführt. Auf der einen Seite argumentieren die Skeptiker mit der Überforderung der Patienten, die Komplexität der medizinischen Behandlung als Laien einschätzen - geschweige denn bewerten - zu können. Auf der anderen Seite gibt es immer mehr Studien, die zeigen, dass Patienten durchaus in der Lage sind, relevante Aspekte der Patientensicherheit zu beobachten und diese auch zu berichten $[1-4]^{*}$. Patienten sind gute Beobachter und an einer sicheren medizinischen Behandlung interessiert. Die von ihnen beobachteten Fehler sind häufig nicht in der Krankenakte oder anderen Unterlagen dokumentiert, obwohl sie retrospektiv klinisch validiert werden können [5, 6]. Deshalb sollten Gesundheitseinrichtungen neben expertenorientierten Ansätzen auch patientenorientierte Ansätze zur Erfassung und Förderung der Patientensicherheit nutzen.

Die Stiftung für Patientensicherheit führte in den Jahren 2008 und 2009 eine Benchmarking-Studie mit acht Deutschschweizer Spitälern durch mit dem Ziel, die von Patienten während des stationären Aufenthalts beobachteten Fehler systematisch zu erfassen und zu analysieren. Die Ergebnisse wurden im Rahmen von Benchmarking-Workshops diskutiert und dienten als Grundlage für die Wahl und Entwicklung von Verbesserungsmassnahmen.

\section{Methode}

Für die schriftliche Befragung von Patienten wurde ein validierter Fragebogen verwendet, der in der Schweiz entwickelt wurde [7]. Acht Spitäler unterschiedlicher

\section{Benchmarking sur la sécurité des patients dans les hôpitaux suisses}

Une étude de benchmarking au sujet des événements qui, du point de vue des patients, relèvent de la sécurité a été réalisée pour la première fois en Suisse. Elle montre que les projets visant à améliorer et promouvoir la sécurité des patients sont utiles et suscitent de l'intérêt. En collaboration avec les hôpitaux de Suisse alémanique, la fondation pour la sécurité des patients a mené une enquête écrite auprès de la patientèle. Les données ainsi recensées ont été analysées dans des workshops de benchmarking et utilisées ensuite pour la mise sur pied de projets d'amélioration internes à l'entreprise. Evaluée sur la base d'entretiens semi-structurés avec les gestionnaires des risques et de la qualité impliqués, cette étude de benchmarking a permis de prouver que les patients représentent une source importante de connaissances pour analyser les données relatives à la sécurité, mais aussi de conclure qu'ils sont à même d'observer les événements importants pour la sécurité. Les institutions du domaine de la santé sont donc appelées à utiliser non seulement l'approche des experts, mais également celle des patients lors de leurs efforts pour garantir la qualité et la sécurité.

Kategorien aus der Deutschschweiz beteiligten sich an der Benchmarking-Studie.

Die Stichprobe umfasste pro Spital 1000 Patienten. Alle stationären Patienten, die während mindestens zwei Nächten stationär behandelt wurden und ein Mindestalter von 18 Jahren hatten, wurden in die Studie eingeschlossen. Jedes Spital versandte nach Aus- 
tritt einen Fragebogen an 500 Patienten der medizinischen Klinik und 500 Patienten der chirurgischen Klink. 10 Tage nach Versand wurde vom Spital ein Erinnerungsschreiben geschickt. Die Befragung war anonym. Die von den Patienten retournierten Fragebögen gingen direkt an die Stiftung für Patientensicherheit.

Die Daten wurden deskriptiv und explorativ ausgewertet. Jedes Spital erhielt die eigenen Ergebnisse, die Ergebnisse der anderen Spitäler und das Total. Mit allen beteiligten Spitälern wurde vereinbart, dass die Ergebnisse zwischen den Studienteilnehmern transparent dargestellt und so für die Benchmarking-Workshops verwendet werden.

Es wurden zwei Benchmarking-Workshops mit Qualitäts- und Risikomanagern der beteiligten Spitäler durchgeführt. Ein erster Workshop diente der Sichtung der Ergebnisse, dem Vergleich der eigenen Ergebnisse der medizinischen und chirurgischen Kliniken und dem Vergleich mit anderen Spitälern. Zudem wurden

\section{Tabelle 1}

«Definitiv» und «möglicherweise» beobachtete sicherheitsrelevante Ereignisse.

\begin{tabular}{|c|c|c|c|}
\hline Item-Nr. & Item & $\begin{array}{l}\text { definitiv } \\
\text { beobachtet } \\
(95 \% \mathrm{Cl})\end{array}$ & $\begin{array}{l}\text { möglicherweise } \\
\text { beobachtet } \\
(95 \% \mathrm{CI})\end{array}$ \\
\hline 1 & $\begin{array}{l}\text { Sie hatten wegen einer Infusion eine Ent- } \\
\text { zündung oder schmerzhafte Rötung der Venen/ } \\
\text { Einstichstelle (Phlebitis) verbunden mit Fieber. }\end{array}$ & $5,2(4,5-5,9)$ & $1,5(1,1-1,9)$ \\
\hline 2 & $\begin{array}{l}\text { Sie haben sich im Spital eine Infektion zuge- } \\
\text { zogen (z. B. Blasenentzündung, Infektion } \\
\text { der Operationsstelle, Blutvergiftung usw.). }\end{array}$ & $4,1(3,5-4.8)$ & $1,1(0,8-14)$ \\
\hline 3 & $\begin{array}{l}\text { Sie haben festgestellt, dass Ärzte oder Pflege- } \\
\text { personen vor einer Handlung an Ihnen } \\
\text { die Hände nicht desinfiziert haben. }\end{array}$ & $3,1(2,6-3,7)$ & $3,9(3,34,5)$ \\
\hline \multirow[t]{2}{*}{4} & Sie haben auf Medikamente allergisch reagiert. & $5,5(4,8-6,3)$ & $1,4(1,1-1,9)$ \\
\hline & $\begin{array}{l}\text { Falls Ja: War Ihre Allergie im Spital vor } \\
\text { der Abgabe des Medikaments bekannt? }\end{array}$ & $13,5(9,1-17,8)$ & $7,1(3,8-10,4)$ \\
\hline 5 & $\begin{array}{l}\text { Sie haben eine Infusion oder ein Medikament } \\
\text { erhalten, die/das nicht für Sie bestimmt war. }\end{array}$ & $0,9(0,6-1,2)$ & $0,5(0,3-0,8)$ \\
\hline \multirow[t]{4}{*}{6} & Sie haben Ihre Medikamente: & & \\
\hline & zum falschen Zeitpunkt & $1,2(0,9-1,6)$ & $0,8(0,6-1,2)$ \\
\hline & in der falschen Dosis erhalten, und/oder & $1,4(1,0-1,8)$ & $1,2(0,9-1,7)$ \\
\hline & eine Dosis wurde aus Versehen vergessen. & $1,5(1,1-2,0)$ & $1,1(0,8-1,4)$ \\
\hline 7 & $\begin{array}{l}\text { Ihre Krankengeschichte oder Röntgenbilder } \\
\text { oder andere wichtige Dokumente waren nicht } \\
\text { verfügbar als sie benötigt wurden. }\end{array}$ & $2,9(2,4-3,5)$ & $2,0(1,6-2,5)$ \\
\hline 8 & $\begin{array}{l}\text { Eine Untersuchung wurde aus Versehen } \\
\text { unnötig wiederholt. }\end{array}$ & $2,1(1,6-2,6)$ & $1,7(1,3-2,1)$ \\
\hline 9 & $\begin{array}{l}\text { Eine geplante Untersuchung wurde } \\
\text { aus Versehen vergessen. }\end{array}$ & $1,2(0,9-1,6)$ & $1,4(1,0-1,8)$ \\
\hline 10 & $\begin{array}{l}\text { Bei Ihnen wurde tatsächlich oder auch } \\
\text { beinahe eine Untersuchung, ein Eingriff oder } \\
\text { eine Therapie an einer falschen Körperstelle } \\
\text { durchgeführt. }\end{array}$ & $0,5(0,3-0,8)$ & $0,4(0,2-0,6)$ \\
\hline 11 & $\begin{array}{l}\text { Sie wurden bei einer Untersuchung oder } \\
\text { Behandlung mit einem anderen Patienten } \\
\text { verwechselt. }\end{array}$ & $0,3(0,2-0,5)$ & $0,2(0,05-0,3)$ \\
\hline 12 & Sie sind im Spital gestürzt. & $2,4(1,9-2,9)$ & $0,2(0,07-0,3)$ \\
\hline
\end{tabular}

erste Erklärungsansätze für Ergebnisunterschiede diskutiert und Prioritäten für spitalindividuelle Verbesserungsmassnahmen gesetzt. Acht Wochen später fand der zweite Workshop statt. In der Zwischenzeit wurden die Ergebnisse den Leitungs- und Entscheidungsgremien in den Spitälern präsentiert. Während des zweiten Workshops berichteten die Teilnehmer über ihre Erfahrungen bei der betriebsinternen Präsentation der Ergebnisse und über weiterentwickelte Erklärungsansätze für die Ergebnisunterschiede und Massnahmenpläne. Ausserdem wurden Ziele für die Umsetzung von Verbesserungsmassnahmen definiert.

Die Zweckmässigkeit der Benchmarking-Studie wurde in semistrukturierten Interviews mit den beteiligten Qualitäts- und Risikomanagern ca. vier Wochen nach dem zweiten Benchmarking-Workshop evaluiert. Die folgenden Fragestellungen wurden erörtert: Nutzbarkeit der Ergebnisse für das Qualitäts- und Risikomanagement, Erkenntnisse zur Umsetzbarkeit der Erhebung und Organisation der Studie, Schlussfolgerungen für betriebsinterne Verbesserungsmassnahmen.

\section{Resultate}

\section{Benchmarking-Studie}

Insgesamt wurden 7254 Fragenbogen versandt, von denen 3983 auswertbar retourniert wurden (Rücklauf $55 \%)$. Der Mittelwert für die Aufenthaltsdauer betrug 9 Tage (95\%, CI=8,8-9,3), und das durchschnittliche Patientenalter lag bei 62,3 Jahren $(95 \%, \mathrm{CI}=61,8-62,9)$.

Ein erheblicher Anteil der Patienten gab an, während des Spitalaufenthaltes um die eigene Sicherheit besorgt gewesen zu sein (3,2\% «sehr besorgt», 14,7\% «etwas besorgt»). Tabelle 1 zeigt die von den Patienten beobachteten sicherheitsrelevanten Ereignisse während ihres stationären Aufenthalts. Die Antwortkategorien beinhalteten neben den Antworten «ja» und «nein» auch die Möglichkeit mit «vielleicht» zu antworten, so dass definitiv und möglicherweise beobachtete Ereignisse berichtet wurden.

21,4\% aller Patienten berichteten mindestens ein Ereignis «definitiv» (291\% mindestens ein Ereignis «möglicherweise» oder «definitiv»). Die durchschnittliche Anzahl berichteter Ereignisse pro Patient liegt bei 0,48 (95\%, CI=0,45-0,51).

Am häufigsten wurden allergische Reaktionen auf verabreichte Medikamente $(5,5 \%, \mathrm{CI}=4,8-6,3)$ berichtet. Von den Patienten, die während ihres Spitalaufenthalts eine allergische Reaktion bei sich beobachteten, gaben 13,5\% (CI=9,1-17,8) an, dass die Allergie dem Spital bekannt gewesen sei.

Am zweithäufigsten $(5,2 \%, \mathrm{CI}=4,5-5,9)$ wurde über eine Entzündung der Venen oder der Einstichstelle berichtet und am dritthäufigsten $(4,1 \%, \mathrm{CI}=3,5-$ 4,8 ) wurden nosokomiale Infektionen (z.B. Blasenentzündung, Infektion der Operationsstelle, Blutvergiftung usw.) beobachtet. Das Ergebnis der entzündeten Einstichstellen bei Venen ist mit Vorsicht zu interpretieren, weil bei dieser Frage nach der Begleiterschei- 
nung des Fiebers gefragt wurde. Innerhalb der Spitäler konnte eine Reihe deutlicher Unterschiede zwischen der Ereignishäufigkeit zwischen den Fachbereichen Medizin und Chirurgie beobachtet werden.

Mittels logistischen Regressionsmodellen wurde geprüft, welche Faktoren das Risiko erhöhen, dass Patienten sicherheitsrelevante Ereignisse berichteten. Dies sind einerseits patientenbezogene Faktoren (z. B. Alter), andererseits auch versorgungsbezogene Faktoren (wie Aufenthaltsdauer). Dabei ist unklar, ob diese Faktoren die tatsächliche Eintrittswahrscheinlichkeit von Ereignissen beeinflussen oder die Berichtswahrscheinlichkeit bei einem eingetretenen Ereignis. Diese Unterscheidung kann aufgrund der vorliegenden Berichtsdaten nicht getroffen werden, zumal diese Wahrscheinlichkeiten interdependent sind. So erhöht die Aufenthaltsdauer vermutlich sowohl das Risiko, dass ein Ereignis eintritt, als auch die Wahrscheinlichkeit, dass eingetretene Ereignisse beobachtet und dadurch auch berichtet werden können. Für die Frage, ob Patienten mindestens eines der abgefragten Ereignisse berichten, sind das Patientenalter $(\mathrm{OR}=0,986$, $\mathrm{CI}=0,98-0,99)$, die Aufenthaltsdauer $(\mathrm{OR}=1,046$, $\mathrm{CI}=1,04-1.06)$, der Gesundheitszustand ( $\mathrm{OR}=0,695$, $\mathrm{CI}=0,63-0,76)$ und das Geschlecht (OR=1,215, CI=1,05-1,4) signifikante Prädiktoren. Die Wahrscheinlichkeit, dass eine 20-jährige Patientin mit schlechtem Gesundheitszustand mindestens ein Ereignis berichtet, liegt bei einer Aufenthaltsdauer von 10 Tagen bei 0,68 (CI=0,61-0,76), während sie bei einem männlichen Patienten im Alter von 70 Jahren mit einem gutem allgemeinen Gesundheitszustand bei $0,17(\mathrm{CI}=0,15-0,19)$ liegt.

\section{Sind Patienten in der Lage, sicherheitsrelevante Ereig- nisse zu beobachten und zu berichten?}

\section{Benchmarking-Workshops}

Die Ergebnisse der Benchmarking-Studie wurden gemeinsam mit den beteiligten Spitälern an zwei Benchmarking-Workshops analysiert und dienten als Grundlage für die Zielsetzung und Planung von Verbesserungsmassnahmen. Tabelle 2 veranschaulicht die aufgrund der Ergebnisse der Benchmarking-Studie von den Qualitäts- und Risikomanagern neu erkannten sicherheitsrelevanten Problemfelder, die in ihren Spitälern prioritär auf der chirurgischen oder medizinischen Klinik behandelt werden müssen.

\section{Evaluations-Interviews}

Die Methode der Benchmarking-Studie und auch die Ergebnisse stiessen auf Interesse bei den Klinikleitungen der beteiligten Spitäler. Nach der Patientenbefragung und den Benchmarking-Workshops wurden die Qualitäts- und Risikomanager zur Nutzbarkeit der Er- gebnisse für betriebsinterne Verbesserungsmassnahmen befragt. Alle beteiligten Qualitäts- und Risikomanager waren sich darüber einig, dass die Benchmarking-Studie für die Förderung der Patientensicherheit nützlich und eine Wiederholung zu einem späteren Zeitpunkt gewünscht ist. Die Nutzbarkeit der Ergebnisse wurde von allen Qualitäts- und Risikomanagern bestätigt und den Ergebnissen wurde betriebsintern Bedeutung seitens der Spitalleitungen beigemessen. Bester Nutzenbeweis ist, dass alle Spitäler wünschten, die Benchmarking-Studie nach einem definierten Zeitraum zu wiederholen.

\section{Diskussion}

Die Ergebnisse der Benchmarking-Studie lieferten wichtige Erkenntnisse und konkrete Ansatzpunkte für Verbesserungsmassnahmen in den Spitälern. So konnten sicherheitsrelevante Ereignisse, die von Patienten beobachtet und berichtet wurden, zur Förderung der Patientensicherheit erhoben und für betriebsinterne Verbesserungsmassnahmen genutzt werden.

Eine solche Studie ist in dieser Form zum ersten Mal in der Schweiz durchgeführt worden. Die Ergebnisse zeigen, dass Patienten durchaus in der Lage sind, sicherheitsrelevante Ereignisse zu beobachten und zu berichten. Patienten stellen damit eine wichtige Wissensressource für die Beurteilung der Patientensicherheit in einer Gesundheitseinrichtung dar.

Da die von Patienten berichteten Ereignisse oftmals nicht in der Patientenakte oder anderen Unterlagen dokumentiert sind $[5,6]$, sollte diesem patientenorientierten Ansatz mehr Aufmerksamkeit geschenkt werden. Die Spitäler gewinnen so wertvolle Informationen, die ihnen sonst nicht zur Verfügung stehen. Der Ansatz stellt in der Diskussion und in den Bemühungen um eine sichere medizinische Behandlung eine neue Dimension dar. Die Ergebnisse zeigen einen deutlichen Handlungsbedarf und motivieren, das Verbesserungspotential auszuschöpfen bzw. Massnahmen zur Reduktion der sicherheitsrelevanten Ereignisse einzuleiten. Sich während des Spitalaufenthaltes sicher fühlende Patienten sind Patienten, die mit der medizinischen Behandlung gesamthaft zufriedener sind [8].

Einige Patienten sind mehr um ihre Sicherheit im Spital besorgt als andere, und auch das Berichten sicherheitsrelevanter Ereignisse ist durch patientenseitige Faktoren beeinflusst. So konnte festgestellt werden, dass jüngere weibliche Patienten mit schlechter allgemeiner Gesundheit eher sicherheitsrelevante Ereignisse berichten als ältere männliche Patienten mit guter Gesundheit. Dieses Ergebnis ist mit Vorsicht zu interpretieren. Jüngere Menschen können zum einen aufmerksamere Beobachter sein und haben vermutlich geringere Hemmungen, kritische Feedbacks zu geben. Patienten mit chronischen Krankheiten sind einerseits einem höheren Risiko für Fehler und Zwischenfälle ausgesetzt und sind andererseits aufgrund ihrer oft langjährigen Erfahrung mit der Gesundheitsversorgung «Experten» und besonders aufmerksame Beob- 
Tabelle 2

Neu erkannte und priorisierte Problemfelder der Spitäler am ersten Benchmarking-Workshop.

\begin{tabular}{|c|c|c|c|c|c|c|c|c|}
\hline Item-Nr. & & Spital A & Spital B & Spital C & Spital D & Spital E & Spital F & Spital G \\
\hline 1 & Phlebitis & Chir.* & & & Chir. & $\begin{array}{c}\text { Chir. } \\
+ \\
\text { Med. }^{* *}\end{array}$ & & \\
\hline 2 & Infektion & & $\begin{array}{c}\text { Chir. } \\
+ \\
\text { Med. }\end{array}$ & $\begin{array}{c}\text { Chir. } \\
+ \\
\text { Med. }\end{array}$ & & & $\begin{array}{c}\text { Chir. } \\
+ \\
\text { Med. }\end{array}$ & Chir. \\
\hline 3 & Händehygiene & $\begin{array}{c}\text { Chir. } \\
+ \\
\text { Med. }\end{array}$ & $\begin{array}{c}\text { Chir. } \\
+ \\
\text { Med. }\end{array}$ & & $\begin{array}{c}\text { Chir. } \\
+ \\
\text { Med. }\end{array}$ & & $\begin{array}{c}\text { Chir. } \\
+ \\
\stackrel{+}{\text { Med. }}\end{array}$ & Chir. \\
\hline 4 & $\begin{array}{l}\text { Allergie auf } \\
\text { Medikament }\end{array}$ & & $\begin{array}{c}\text { Chir. } \\
+ \\
\text { Med. }\end{array}$ & Chir. & Med. & & $\begin{array}{c}\text { Chir. } \\
+ \\
\text { Med. }\end{array}$ & Chir. \\
\hline 5 & $\begin{array}{l}\text { Medikamenten- } \\
\text { verwechslung }\end{array}$ & & $\begin{array}{c}\text { Chir. } \\
+ \\
\text { Med. }\end{array}$ & Chir. & Med. & & $\begin{array}{c}\text { Chir. } \\
+ \\
\text { Med. }\end{array}$ & Chir. \\
\hline 6 & Medikationsfehler & & $\begin{array}{c}\text { Chir. } \\
+ \\
\text { Med. }\end{array}$ & Chir. & Med. & $\begin{array}{c}\text { Chir. } \\
+ \\
\text { Med. }\end{array}$ & & Chir. \\
\hline 7 & $\begin{array}{l}\text { Dokumentation } \\
\text { nicht verfügbar }\end{array}$ & & & & Med. & & & $\begin{array}{c}\text { Chir. } \\
+ \\
\text { Med. }\end{array}$ \\
\hline 8 & $\begin{array}{l}\text { Untersuchung un- } \\
\text { nötig wiederholt }\end{array}$ & & & Chir. & Med. & & & \\
\hline 9 & $\begin{array}{l}\text { Untersuchung } \\
\text { vergessen }\end{array}$ & & & Chir. & & & & Chir. \\
\hline 10 & Falsche Körperstelle & Med. & & & & & & \\
\hline 11 & $\begin{array}{l}\text { Patienten- } \\
\text { verwechslung }\end{array}$ & Med. & & & & & & \\
\hline \multirow[t]{3}{*}{12} & Sturz & & $\begin{array}{c}\text { Chir. } \\
+ \\
\text { Med. }\end{array}$ & Med. & & & Med. & Chir. \\
\hline & Kommunikation & $\begin{array}{c}\text { Chir. } \\
+ \\
\text { Med. }\end{array}$ & & Chir. & Med. & $\begin{array}{c}\text { Chir. } \\
+ \\
\text { Med. }\end{array}$ & Med. & $\begin{array}{c}\text { Chir. } \\
+ \\
\text { Med. }\end{array}$ \\
\hline & Besorgnis & & & Chir. & & $\begin{array}{c}\text { Chir. } \\
+ \\
\text { Med. }\end{array}$ & & Med. \\
\hline
\end{tabular}

achter. Aufgrund dieser Einflussfaktoren wird die Verteilung der genannten Merkmale in den Patientenkollektiven der beteiligten Spitäler immer einen Einfluss auf die Häufigkeit der berichteten Ereignisse haben. Entscheidend bei der Interpretation der Ergebnisse ist, dass nicht primär der Vergleich mit den Ergebnissen anderer Spitäler für die Initialisierung von Verbesserungsmassnahmen ausschlaggebend sein darf, sondern die Ergebnisse jedes einzelnen Spitals mit den betriebsinternen Fachleuten diskutiert und entsprechende individuelle Zielvereinbarungen getroffen werden müssen. Solche Zielsetzungen können die spezifische Situation und die Umwelt des Spitals berücksichtigen.

Die Ergebnisse der Studie sind durch einige Faktoren limitiert. In der Kürze der Zeit zwischen erstem und zweitem Benchmarking-Workshop war es nicht möglich, Verbesserungsmassnahmen so wirksam zu implementieren, dass deren Nachhaltigkeit evaluiert werden könnte. Die Evaluation durch die beteiligten
Spitäler zeigte jedoch, dass den Ergebnissen eine Bedeutung auch auf Ebene der Spitalleitungen beigemessen wurde. Für zukünftige Studien wird über den Einsatz des Patienten-Fragebogens hinaus eine Kombination von verschiedenen Datenquellen und Perspektiven (patienten- und expertenorientierter Ansatz) interessant sein.

\section{Danksagung}

Die Stiftung für Patientensicherheit bedankt sich für das Engagement der folgenden Qualitäts- und Risikomanager, die sich an der Benchmarking-Studie beteiligt und der Publikation der Ergebnisse zugestimmt haben: Ute Buschmann (Luzerner Kantonsspital), Karin Aeberhard (Spitalzentrum Biel AG), Carmela Fornasier (Klinik Lindberg), Paul Geiser (Spital Zofingen), Fritz Iff (ehemals Stadtspital Triemli Zürich), Kerstin Moeller (Spital Schwyz), Rolf Prions (ehemals Kantonsspital Uri), Heiner Zurbrügg (Spital STS AG). 


\section{Literatur}

1 Wolosin RJ, Vercler L, Matthews KL. Am I safe here? Improving patients' perceptions of safety in hospitals. J Nurs Care Qual. 2006;21:30-8.

2 Fowler FJ, Epstein A, Weingart SN, Annas CL, Bolcic-Jankovic D, Clarridge B et al. Adverse events during hospitalization: results of a patient survey. Jt Comm J Qual Patient Saf. 2008;34:583-90.

3 Weingart SN, Pagovich O, Sands DZ, Li JM, Aronson MD, Davis RB et al. What can hospitalized patients tell us about adverse events? Learning from patient-reported incidents. J Gen Intern Med. 2005;20:830-6.

4 Cleary PD, Edgman-Levitan S, Roberts M, Moloney TW, McMullen W, Walker JD et al. Patients evaluate their hospital care: a national survey. Health Affairs. 1991;10(4):254-67.

5 Weissmann JS, Schneider EC, Weingart SN, Epstein AM, David-Kasdan J, Feibelman S et al. Comparing patient-reported hospital adverse events with medical record review: do patients know something that hospitals do not? Ann Intern Med. 2008;149:100-8.

6 Fränneby U, Sandblom G, Nyren O, Nordin P, Gunnarsson U. Self-reported adverse events after groin hernia repair. A study based on a national register. Value Health. 2008;11:927-32.

7 Schwappach DL. Against the silence: Development and first results of a patient survey to assess experiences of safety-related events in hospital. BMC Health Serv Res. 2008;8:59.

8 Burroughs TE, Waterman AD, Gallagher TH, Waterman B, Jeffe DB, Dunagan WC et al. Medication Safety: Patients' concerns about medical errors during hospitalization. Jt Comm J Qual Pat Saf. 2007;33:5-14. 